\title{
Geometry for contouring by electronic speckle pattern interferometry based on shifting illumination beams
}

\author{
Y. Zou, H. Diao, X. Peng, and H. Tiziani
}

\begin{abstract}
When the method of contouring an object surface by electronic speckle pattern interferometry is based on shifting the illumination beams, the shifted phase of the interference speckle pattern has a new relationship with the depth of the test surface. Therefore the contour interval as well as the fringe sensitivity of this method has new forms. The geometry of such a situation, which differs from that of either the method of two-wavelength contouring or the method of contouring by tilting the test object is presented. The requirements on the experimental conditions for this method are also presented. Experimental results are in agreement with these analyses.

Key words: Interferometry, speckle, speckle interferometry
\end{abstract}

\section{Introduction}

Optical contouring techniques applied on a rough surface by means of electronic speckle pattern interferometry (ESPI) have been known for a long time. They provide data for three-dimensional shape analysis and topography measurement. The advantages of employing ESPI is that intermediate photographic processing is eliminated and a precise replica of the original test object, which is often critical with holographic contouring, is unnecessary. The real-time correlation fringes that correspond to the shape depth of the test surface are shown on a monitor. Furthermore it is possible to use a host computer directly to analyze the live fringes. The basic theories of ESPI have been discussed in the literature. ${ }^{1,2}$

Several methods of contouring by ESPI have been developed. The classical method employes two wavelengths for illumination. The contour interval is $d=\Lambda_{12}$, where $\Lambda_{12}$ is the equivalent wavelength of the two wavelengths, and the fringe sensitivity can be varied by altering the difference between the two wavelengths. ${ }^{2}$ Another method involves deformation of the test object and using dual-beam illumination $^{3}$ to obtain correlation fringes. The contour interval in this case is $d=\lambda /\left(2 \phi_{t} \sin \theta\right)$, where $\lambda$ is the wavelength of the illumination, $\phi_{t}$ is the tilt of the

The authors are with the Institute of Applied Optics, University of Stuttgart, Pfaffenwaldring 9, Stuttgart D-7000 80, Germany.

Received 27 August 1991.

0003-6935/92/316616-06\$05.00/0.

(C) 1992 Optical Society of America. object, and $\theta$ is the illumination angle. The sensitivity is determined by tilting the object or altering the angle of illumination. Recently a new method has been reported that involves shifting the illumination beams in the case of either one illumination beam with one reference beam ${ }^{4,5}$ or dual-beam illumination $^{6,7}$ instead of tilting the test object. The contour intervals are $d=\lambda / \delta \theta$ for the first case with an illumination angle of $90^{\circ}$ and $d=\lambda /(2 \delta \theta \sin \theta)$ for the second case, where $\delta \theta$ is the angle change of the illumination beams and $\theta$ is the illumination angle. The fringe sensitivity can now be controlled easily by altering the scanning angle $\delta \theta$ of the illumination beams when the illumination angle $\theta$ is at a certain value. The possibility of identifying a stationary reference contour and the possibility of programmable control of sensitivity are attractive features of the latter methods.

The complete geometry of the method of contouring by shifting illumination beams is presented in this paper. The fringe sensitivity and the requirements of the experimental conditions of the method are also discussed. The experimental results by a dual-beam ESPI based on shifting illumination beams correlate well with our analysis. However, the theory would be valid for any method involving changes in the illumination beams.

\section{Basic Theories}

With ESPI an image of an object is formed on a TV or CCD camera; the object is illuminated by coherent light. In a dual-beam case the object is illuminated 
by two beams so that the intensity of the speckle pattern formed on the image is a function of the phase as well as of the amplitude of the light scattered from the object.

As in Fig. 1 the complex amplitudes of both illumination beams scattered from a point $M$ on the object can be expressed at a given point $P$ on the image as

$$
\begin{aligned}
& \tilde{U}_{1}(\mathrm{P})=A_{s 1} \exp i\left(\phi_{s 1}+\phi_{m 1}\right), \\
& \tilde{U}_{2}(\mathrm{P})=A_{s 2} \exp i\left(\phi_{s 2}+\phi_{m 2}\right),
\end{aligned}
$$

respectively, where $A_{s 1}, A_{s 2}$ and $\phi_{s 1}, \phi_{s 2}$ are the random amplitudes and phases associated with the speckle obtained from the conjugate point of the object, and $\phi_{m 1}, \phi_{m 2}$ are phases associated with the optical path from point $M$ to point $P$. The intensity at the point $\mathrm{P}$ is then given by

$$
\Gamma_{1}(\mathrm{P})=I_{1}^{2}+I_{2}^{2}+2\left(I_{1} I_{2}\right)^{1 / 2} \cos \left[\left(\phi_{s 1}-\phi_{s 2}\right)+\Psi_{m}\right],
$$

and

$$
\begin{aligned}
I_{1} & =\tilde{U}_{1} \tilde{U}_{1}{ }^{*}, \\
I_{2} & =\tilde{U}_{2} \tilde{U}_{2}{ }^{*}, \\
\Psi_{m} & =\phi_{m 1}-\phi_{m 2} .
\end{aligned}
$$

If the phases $\phi_{m 1}$ and $\phi_{m 2}$ are changed to $\phi_{m 1}{ }^{\prime}$ and $\phi_{m 2}$ ' by undergoing some process, for example, a shift of the illumination beams, the intensity at point $P$ will be changed to $\Gamma_{2}$ where

$$
\begin{aligned}
& \Gamma_{2}(\mathrm{P})=I_{1}^{2}+I_{2}^{2}+2\left(I_{1} I_{2}\right)^{1 / 2} \cos \left[\left(\phi_{s 1}-\phi_{s 2}\right)+\Psi_{m}{ }^{\prime}\right] \\
& \Psi_{m}{ }^{\prime}=\phi_{m 1}{ }^{\prime}-\phi_{m 2}{ }^{\prime} .
\end{aligned}
$$

The phasor amplitude of the electric field at viewpoint
$\mathrm{P}$ is a sum of a number $(\mathrm{N})$ of dephased elementary phasor contributions from different scattering regions of the rough surface. ${ }^{8}$ If the illumination shifting is not significantly large, the number of changed elementary phasors is much smaller than the sum number $N$. Therefore the random amplitudes and random phases are assumed to be the same in Eqs. (2) and (3). If not, a decorrelation effect will appear (see Ref. 2, Appendix F).

The correlation between intensities $\Gamma_{1}$ and $\Gamma_{2}$ can be observed by video signal subtraction or addition. In the subtraction process the camera video signal corresponding to the interferometer image plane speckle pattern $\Gamma_{1}$ is stored electronically. The video signal is then digitized and stored by a frame buffer and a host computer. The live video signal of $\Gamma_{2}$, as detected also by the camera, is subtracted from the stored wave form. The output is then displayed on a TV monitor where the correlation fringes may be observed live. The output video signals from the camera $V_{1}$ and $V_{2}$ are proportional to the input image intensities, then the subtracted signal is given by

$$
\begin{aligned}
V_{s}= & \left(V_{1}-V_{2}\right) \propto\left(\Gamma_{1}-\Gamma_{2}\right) \\
= & 4\left(I_{1} I_{2}\right)^{1 / 2} \sin \left[\left(\phi_{s 1}-\phi_{s 2}\right)+\left(\Psi_{m}+\Psi_{m}{ }^{\prime}\right) / 2\right] \\
& \times \sin \left(\Delta \Psi_{m} / 2\right),
\end{aligned}
$$

where $\Delta \Psi_{m}=\Psi_{m}{ }^{\prime}-\Psi_{m}$

The brightness $B$ of a TV monitor is defined as ${ }^{1}$

$$
B \propto\left\langle\left(V_{1}-V_{2}\right)^{2}\right\rangle^{1 / 2} .
$$

After averaging and bandpass filtering, the random term containing $\phi_{s 1}$ and $\phi_{s 2}$ in Eq. (4) becomes uniform with a value of 1 . Then

$$
B \propto\left(I_{1} I_{2} \sin ^{2} \frac{\Delta \Psi_{m}}{2}\right)^{1 / 2}
$$

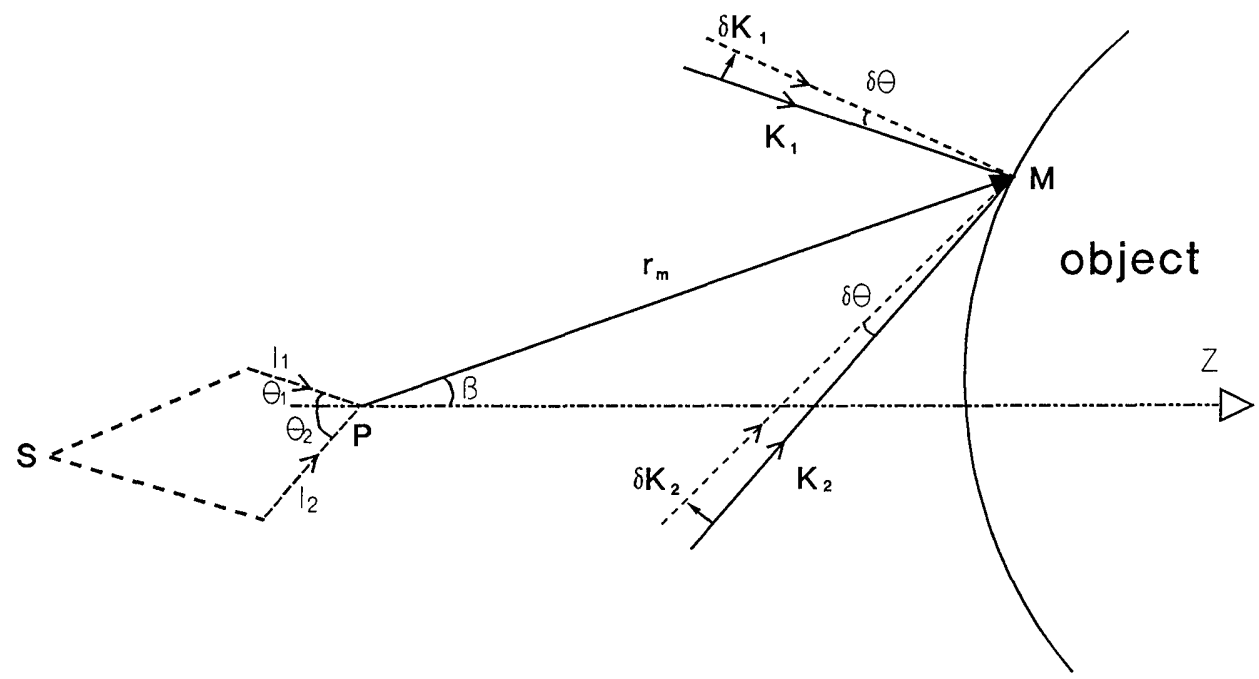

Fig. 1. Geometry of dual-beam-shifted ESPI contouring. 
Dark correlation fringes appear when $\Delta \Psi_{m}=2 n \pi$, where $n$ is any integer.

\section{Geometric Analysis}

From Eq. (6) we can see that the fringe interval is determined only by the phase difference between those two interference speckle patterns. When this phase difference is related to the depth of the object, the shape of the object can be obtained from the fringe pattern interpretation. In the following we give a complete geometric analysis of the relationship between the phase difference and the depth of the object in the contouring by a dual-beam ESPI based on shifting illumination beams.

The object is illuminated by two plane waves from both sides about the view direction $Z . \quad \mathbf{K}_{1}$ and $\mathbf{K}_{2}$ are the unit direction vectors of these two waves, respectively. The geometry is shown in Fig. 1. $\mathrm{M}$ is an arbitrary point on the test surface, and $\mathrm{P}$ is the viewpoint; $\mathbf{r}_{m}$ is the position vector from point $P$ to $M$. $\beta$ is the angle between $\mathbf{r}_{m}$ and the $Z$ axis. $\delta \mathbf{K}_{1}$ and $\delta \mathbf{K}_{2}$ are the direction changes of $\mathbf{K}_{1}$ and $\mathbf{K}_{2}$, respectively, after the first speckle pattern is stored. And $\delta \theta$ is the small scanning angle of beam $\mathbf{K}_{1}$ or $\mathbf{K}_{2}$ when the illumination beams are shifted by the same amount $\left(\left|\delta \mathbf{K}_{1}\right|=\left|\delta \mathbf{K}_{2}\right|=\delta \theta\left|\mathbf{K}_{1}\right|=\delta \theta\left|\mathbf{K}_{2}\right|=\delta \theta\right.$, where $\left|\mathbf{K}_{1}\right|=\left|\mathbf{K}_{2}\right|=1$; see Fig. 1). If the optical paths of the two illumination beams directly from the source to viewpoint $\mathrm{P}$ are $l_{1}$ and $l_{2}$, respectively, the phase advances of the light scattered from point $M$ to $P$ are given as

$$
\begin{aligned}
& \phi_{m 1}=\frac{2 \pi}{\lambda}\left(r_{m}+l_{1}+\mathbf{r}_{m} \cdot \mathbf{K}_{1}\right), \\
& \phi_{m 2}=\frac{2 \pi}{\lambda}\left(r_{m}+l_{2}+\mathbf{r}_{m} \cdot \mathbf{K}_{2}\right),
\end{aligned}
$$

respectively, where $r_{m}$ is the scalar magnitude of $\mathbf{r}_{m}$. The phase of the interference speckle pattern $\Psi_{m}$ then has a form [see Eq. (2)]

$$
\Psi_{m}=\frac{2 \pi}{\lambda}\left[\left(l_{1}-l_{2}\right)+\mathbf{r}_{m} \cdot\left(\mathbf{K}_{1}-\mathbf{K}_{2}\right)\right] .
$$

When the phase difference between those two speckle patterns is relatively small compared with the phase itself, the difference in the phase can be considered as just a variation of the phase. Therefore

$$
\begin{aligned}
\delta \Psi_{m} & =\frac{2 \pi}{\lambda} \mathbf{r}_{m} \cdot\left(\delta \mathbf{K}_{1}-\delta \mathbf{K}_{2}\right) \\
& =\frac{2 \pi}{\lambda}\left|\mathbf{r}_{m}\right|\left|\delta \mathbf{K}_{1}-\delta \mathbf{K}_{2}\right| \cos (\beta-\alpha) \\
& =\frac{2 \pi}{\lambda} r_{m} 2 \sin \left(\frac{\theta_{1}+\theta_{2}}{2}\right) \delta \theta \cos (\beta-\alpha) \\
& =\frac{2 \pi}{\lambda} 2 \sin \left(\frac{\theta_{1}+\theta_{2}}{2}\right) \delta \theta h,
\end{aligned}
$$

$$
h=r_{m} \cos (\beta-\alpha),
$$

where $\mathbf{r}_{m}$ is fixed, $\left|\mathbf{r}_{m}\right|=r_{m},\left(l_{1}-l_{2}\right)$ is assumed to be constant during the scanning of the illumination beams, for example, when $l_{1}=l_{2}$ (see Section 4$)$,

$$
\left|\delta \mathbf{K}_{1}-\delta \mathbf{K}_{2}\right|=2 \sin \left(\frac{\theta_{1}+\theta_{2}}{2}\right) \delta \theta,
$$

and $\alpha$ is the angle between the vector $\left(\delta \mathbf{K}_{1}-\delta \mathbf{K}_{2}\right)$ and the $Z$ axis (see Fig. 2a), and

$$
\alpha=\frac{\theta_{2}-\theta_{1}}{2}
$$

(see Fig. 2b).

If the directions of the two illuminations are symmetric about the view direction, i.e., $\theta_{1}=\theta_{2}$, we have $\alpha=0$. Then $h=r_{m} \cos \beta$. Now $h$ represents the depth of the object along the view direction $Z$. Therefore the difference in depth along this direction can be mapped out by the correlation fringes. The contour interval (i.e., the difference between two adjacent fringes) is then as follows:

$$
d=\frac{\lambda}{2 \sin \theta \delta \theta}
$$

Obviously the sensitivity can be controlled by altering the shifting angle $\delta \theta$ and the illumination angle $\theta$.

An experimental example is shown in Fig. 3. A small scan of both illumination beams on the object is introduced by shifting the collimating lens $L$ in the perpendicular direction to the axis. Mirror $\mathrm{M}_{3}$ plays the role of a direction compensator to keep the scanning of $K_{1}$ and $K_{2}$ in the same direction. Equations (9) and (10) are the result. The test object is a pyramid with an apex angle of $120^{\circ}$ whose center axis is normal to the image plane of the camera. A He-Ne laser with a 633-nm spectral line is used as a coherent source. A contour fringe pattern with $\theta=30^{\circ}$ and $\delta \theta=1.3 \mathrm{mrad}$ is shown in Fig. 4.
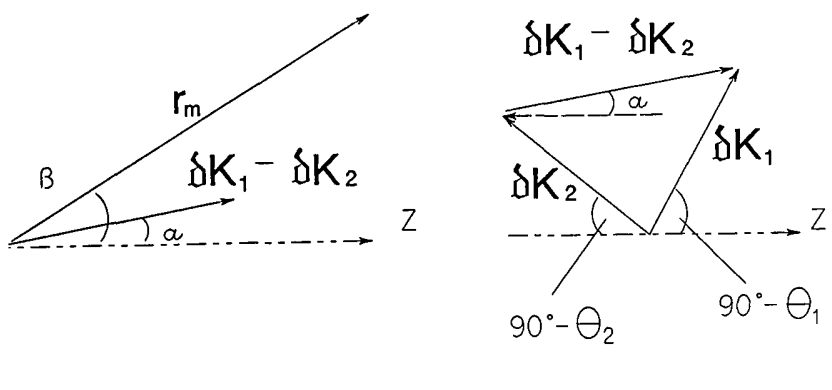

a

b

Fig. 2. Vector geometry of the contouring. 


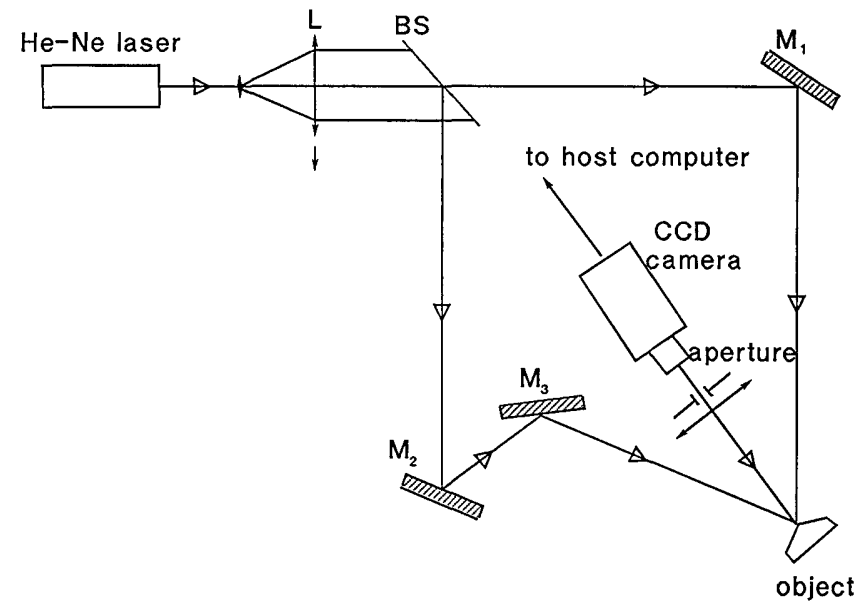

Fig. 3. Experimental arrangement for dual-beam-shifted ESPI contouring.

\section{Several cases result from this example.}

Case 1: Only one illumination beam is shifted for the second speckle pattern. For example, only $\mathbf{K}_{1}$ shifts in Fig. 1. Then $\delta \mathbf{K}_{2}=0$, and

$$
\begin{aligned}
\delta \Psi_{m} & =\frac{2 \pi}{\lambda} \mathbf{r}_{m} \cdot \delta \mathbf{K}_{1} \\
& =\frac{2 \pi}{\lambda}\left|\mathbf{r}_{m}\right|\left|\delta \mathbf{K}_{1}\right| \cos \left(90^{\circ}-\theta_{1}-\beta\right) \\
& =\frac{2 \pi}{\lambda} r_{m} \delta \theta \sin \left(\theta_{1}+\beta\right) \\
& =\frac{2 \pi}{\lambda} \delta \theta h,
\end{aligned}
$$

where $\left|\mathbf{r}_{m}\right|=r_{m},\left|\delta \mathbf{K}_{1}\right|=\delta \theta$, and

$$
h=r_{m} \sin \left(\theta_{1}+\beta\right) \text {. }
$$

Obviously $h$ no longer represents the depth of the s." :



Fig. 4. Contour fringes of a pyramid with $\theta=30^{\circ}, \delta \theta=1.3 \mathrm{mrad}$, and $d=0.49 \mathrm{~mm}$. object along the view direction $Z$ [compared with Eq. (10)]. The correlation fringes do not correspond directly to the height of the object in the view direction. Therefore a computer fit to a polynomial function must be used to obtain a contour plot.5,7 For the contour planes normal to the $Z$ axis $\theta_{1}$ must be equal to $90^{\circ}$, and hence

$$
h=r_{m} \cos \beta .
$$

This indicates that the shifted illumination beam should be approaching a grazing incident angle. This effect is independent of the incident angle of another illumination beam. The contour interval is therefore

$$
d=\frac{\lambda}{\delta \theta}
$$

The sensitivity is then determined only by $\delta \theta$. This is quite easily proved by using the setup in Fig. 3 with a slight change in $\theta$ (see also Ref. 4).

Case 2. Both illumination beams are shifted as by rotation about two separate axes in opposite directions.

If mirror $M_{3}$ in Fig. 3 is eliminated and the second illumination beam comes directly from $\mathrm{M}_{2}$, these two beams scan the object in opposite directions when the lens $\mathrm{L}$ is shifted. In this case we have

$$
\alpha=90^{\circ}+\frac{\theta_{1}-\theta_{2}}{2} .
$$

When $\theta_{1}=\theta_{2}$, from Eq. (9) we obtain $h=r_{m} \sin \beta$. This indicates that the contour planes are perpendicular to the plane determined by the $Z$ axis. Now it is no longer convenient to analyze the fringe pattern from the view direction.

\section{Requirements of Experimental Conditions}

Several special experimental conditions need to be identified for the case where the fringes that are obtained from ESPI based on shifting illumination beams actually contour the surface. First, to obtain Eq. (9) we have assumed that the optical length difference between the two illumination beams $\left(l_{1}-l_{2}\right)$ was not changed by shifting the beams. If this term were changed, we have

$$
\begin{aligned}
\delta \Psi_{m}= & \frac{2 \pi}{\lambda}\left[\delta\left(l_{1}-l_{2}\right)+2 h \sin \left(\frac{\theta_{1}+\theta_{2}}{2}\right) \delta \theta\right] \\
& \simeq \frac{2 \pi}{\lambda}\left[\frac{l_{2}-l_{1}}{2}(\delta \theta)^{2}+2 h \sin \left(\frac{\theta_{1}+\theta_{2}}{2}\right) \delta \theta\right] .
\end{aligned}
$$

The second order of the shifted angle $\delta \theta$ appears in the phase term (see Appendix A). To avoid it, one can let $l_{1} \simeq l_{2}$. This is also the best condition for obtaining good interference between two illuminations.

Other critical requirements are as follows: letting 
plane $\delta \mathbf{K}_{1} \times \delta \mathbf{K}_{2}$ be in plane $\mathbf{K}_{1} \times \mathbf{K}_{2}$, choosing a diffraction-limited collimation lens $L$ (if we do not intentionally choose à relative small lens, the decorrelation effect will appear when the shifting of the collimating lens is efficiently great ${ }^{9}$ before aberrations start affecting the contour fringes), and setting the two illumination beams to overlay exactly on the test surface.

When any of these conditions is not satisfied, error fringes occur.

\section{Discussion}

The phase contours of the test surface can be determined by phase-shifting techniques from the correlation fringe pattern. ${ }^{6}$ The sensitivity and the measurement accuracy of this method depend on the accuracy in measuring the scanning angle $\delta \theta$ and the illumination angle $\theta$. It is quite easy to obtain a high sensitivity for steep objects and a great sensitivity range by only altering the scanning angle $\delta \dot{\theta}$. The collimating lens $\mathrm{L}$ could be mounted on a microdriver for this purpose.

The fringe visibility or contrast in this method depends on the beam ratio of the two illumination beams, the total intensity of the image on the CCD camera, the correct imaging relationship between the object surface and the camera, the speckle size, and the decorrelation effect in the system. The optimum condition can be achieved through experimentation.

The contour principle with shifted illumination beams, which differs from that of the two-wavelength contour method and the tilted object contour method, was demonstrated in this paper. The theory is based on vector analysis. The conditions of one shifted illumination and of two illuminations with different shifting directions have been discussed. The locations of the contour planes and the sensitivities differ from one case to another. This analysis is not only useful for the experiments with shifting illumination beams, but it is also valid for any method in which two speckle patterns at two different conditions are subtracted from each other in ESPI.

\section{Appendix A: Influence of the Second Order of $\delta \theta$ on $\delta \Psi_{m}$}

In our setup scanning the illumination beams was performed by shifting the collimating lens L (see Fig.
3). The optical paths before and after shifting $L$ differ from each other. As in Fig. 5 light from the source $S$ to the viewpoint $P$ for one illumination beam undergoes lengths $l_{1}$ and $l_{1}{ }^{\prime}$ before and after a lens shifting of the order of $\Delta X$ in the $X$ direction. The phase from $\mathrm{S}$ to wave front $\mathrm{C}$ before lens $\mathrm{L}$ is shifted or from $\mathrm{S}$ to $\mathrm{C}^{\prime}$ after the lens is shifted is constant. Therefore $l_{1}=\overline{\mathrm{SP}}, l_{1}{ }^{\prime}=\overline{\mathrm{VP}}=\overline{\mathrm{SQ}}$. From simple geometry, we have

$$
l_{1}^{\prime}=l_{1} \cos \delta \theta
$$

then

$$
\begin{aligned}
\Delta l_{1}= & l_{1}{ }^{\prime}-l_{1}=-2 l_{1} \sin ^{2}\left(\frac{\delta \theta}{2}\right) \\
& \simeq-\frac{l_{1}}{2}(\delta \theta)^{2} \quad(\delta \theta \ll 1) .
\end{aligned}
$$

Similarly,

$$
\Delta l_{2}=-\frac{l_{2}}{2}(\delta \theta)^{2}
$$

Thus

$$
\Delta\left(l_{1}-l_{2}\right)=\frac{l_{2}-l_{1}}{2}(\delta \theta)^{2} .
$$

We obtain Eq. (17) from Eqs. (8) and (21).

This analysis is valid with only one prerequisite: The lens $L$ is a thin lens, and all activities occur only near the axis of the lens.

A typical set of experimental data is as follows: $\lambda=0.633 \mu \mathrm{m}, l=l_{1}=l_{2}=180 \mathrm{~cm}, \theta=30^{\circ}$, and $\delta \theta=$ $1.3 \mathrm{mrad}$. If $\Delta l=l_{2}-l_{1}=5 \mathrm{~cm}$, from Eq. (17) we have the influence of the second order of $\delta \theta$ on $\delta \Psi_{m}$ of $\sim 0.42 \mathrm{rad}$. This is $\sim 6.7 \%$ of a shifted fringe. It changes the absolute data of the phase, but the contour interval is the same as in Eq. (11), because the term of this second order of $\delta \theta$ in Eq. (17) is independent of the fringe orders $n$ where $\delta \Psi_{m}=2 n \pi$.

$\mathrm{X}$. Peng, an Alexander von Humboldt research fellow, thanks the Alexander von Humboldt Foundation for financial support.



Fig. 5. Geometry for the analysis of the second order of $\delta \theta$. 


\section{References}

1. J. N. Butters, R. Jones, and C. Wykes, "Electronic speckle pattern interferometry," in Speckle Metrology, R. K. Erf, ed. (Academic, New York, 1978), Chap. 6, pp. 111-158.

2. R. Jones and C. Wykes, Holographic and Speckle Interferometry (Cambridge U. Press, Cambridge, 1989), Chap. 4, p. 165; Chap. 5, p. 197.

3. C. Joenathan, B. Pfister, and H. Tiziani, "Contouring by electronic speckle pattern interferometry employing dual beam illumination," Appl. Opt. 29, 1905-1911 (1990).

4. B. D. Bergquist and P. Montgomery, "Contouring by electronic speckle pattern interferometry," in Optics in Engineering Measurement, W. F. Fagan, ed., Proc. Soc. Photo-Opt.. Instrum. Eng. 599, 189-195 (1985).

5. S. Winther and G. Slettemoen, "An ESPI contouring technique in strain analysis," in Symposium Optika '84, G. Luprovics and
A. Podmaniczby, eds., Proc. Soc. Photo-Opt. Instrum. Eng. 473, 44-47 (1984).

6. X. Peng, H. Y. Diao, Y. L. Zou, and H. Tiziani, "Contouring by modified dual-beam ESPI based on tilting illumination beams," Optik (Stuttgart) 90, 61-64 (1992).

7. D. Kerr, R. R. Vera, and F. M. Santoyo, "Surface contouring using electronic speckle pattern interferometry," in Second International Conference on Photomechanics and Speckle Metrology, F.-P. Chiang, ed., Proc. Soc. Photo-Opt. Instrum. Eng. 1554A, 668-679 (1991).

8. J. W. Goodman, "Statistical properties of laser speckle patterns," in Laser Speckle and Related Phenomena, J. C. Dainty, ed. (Springer-Verlag, Berlin, 1975), Chap. 2, pp. 12-14.

9. X. Peng, H. Y. Diao, Y. L. Zou, and H. Tiziani, "A novel approach to determine decorrelation effect in a dual-beam electronic speckle pattern interferometer," to be published in Optik (Stuttgart) 90, 129-133 (1992). 